\title{
Modeling of Carrier Transport Dynamics at GHz-Frequencies for RF Circuit-Simulation
}

\author{
D. Navarro, N. Nakayama ${ }^{1}$, K. Machida ${ }^{2}$, Y. Takeda, S. Chiba, \\ H. Ueno, H. J. Mattausch, M. Miura-Mattausch, T. Ohguro ${ }^{1}$, \\ T. Iizuka ${ }^{1}$, M. Taguchi ${ }^{1}$, and S. Miyamoto ${ }^{1}$ \\ Graduate School of Advanced Sciences of Matter, Hiroshima University \\ 1-3-1, Kagamiyama, Higashi-Hiroshima, 739-8530, Japan \\ E-mail: navarro@hiroshima-u.ac.jp \\ ${ }^{1}$ Semiconductor Technology Academic Research Center, Yokohama, Japan \\ ${ }^{2}$ Mathematical Systems, Inc., Tokyo, Japan
}

\begin{abstract}
Carrier dynamics in a MOSFET channel under fast time-varying gate input is included in the modeling for circuit simulation and implemented in SPICE3f5 at only 7\% increased computational runtime cost. Correct reproduction of transient drain currents as well as harmonic-distortion characteristics are verified. While the carrier dynamics under low-frequency operation is mostly governed by the carrier mobility in the channel, the dominant factor under high-frequency operation changes to channel charging and discharging.
\end{abstract}

\section{Introduction}

With recent developments of integrating scaled-down MOSFETs in RF technology applications, the traditional description of the carrier dynamics in the channel becomes inadequate due to high-frequency-related phenomena. One observation is the gate capacitance reduction which becomes very large near the cut-off frequency region as shown in Fig. 1. This phenomenon is explained by the fact that carriers do not respond instantaneously to very fast switching and must be modeled accurately. The delayed carrier response also manifests as a delay in the formation of the drain current as shown in the transient simulation of Fig. 2. Another phenomenon, important for RF applications, is harmonic distortion (HD), which results from the non-linear $I-V$ characteristics of the MOSFET device [1]. An input of a single fundamental frequency $\omega_{0}$ produces harmonics in the output as depicted in Fig. 3. HD has been investigated up to $1 \mathrm{GHz}$ by dividing the MOSFET into multiple segments [2]. However, an accurate physical analysis for high-frequency operation is still missing. Correct prediction of HD is one of the most sensitive indicators of model accuracy [3], and requires a physical description of the dynamics of carriers in the channel.

Here we develop a non-quasi-static (NQS) model which reproduces the changes in the device characteristics at fast switching. The model is particularly important to secure reliable circuit-simulation of RF-devices. Furthermore, the dynamics of carriers via $\mathrm{HD}$ for a pocket-implant technology with $1.2 \mathrm{~V}$ supply voltage is investigated using the developed model. 


\section{Modeling Based on Delayed Carrier Response}

Under fast switching, the time it takes to build up carriers in the channel must be considered as opposed to the conventional quasi-static (QS) approach. For this purpose, the carrier formation in the channel is modeled with a transit delay $\tau$ as

$q\left(t_{\mathrm{i}}\right)=q\left(t_{\mathrm{i}-1}\right)+\frac{t_{\mathrm{i}}-t_{\mathrm{i}-1}}{\tau}\left(Q\left(t_{\mathrm{i}}\right)-q\left(t_{\mathrm{i}-1}\right)\right)$

where $q\left(t_{\mathrm{i}}\right)$ and $Q\left(t_{\mathrm{i}}\right)$ represent transient carrier density and quasi-static carrier density at time $t_{\mathrm{i}}$, respectively. $Q\left(t_{\mathrm{i}}\right)$ is calculated by the QS model HiSIM (Hiroshimauniversity STARC IGFET Model) [4] based on the channel-surface potential. $t_{\mathrm{i}-1}$ indicates quantity values at the previous time step. The equation implies that the formation of carriers is always delayed by $\tau$ with respect to the QS case. $\tau$ consistently includes the delay mechanisms determining the channel-charge formation. At weak inversion, $\tau$ is governed by diffusion. At strong inversion, the delay to form the channel is determined by the carrier velocity, which is calculated directly from the quasi-static model. These delay mechanisms are combined using the Matthiessen rule. Delay mechanisms in MOSFET saturation $\left(V_{\mathrm{gs}}=1 \mathrm{~V}\right)$ for a gate-voltage rise time $t_{\mathrm{r}}$ of 20ps are shown in Fig. 4.

\section{Results and Discussion}

Equation 1, with $\tau$ dynamically determined according to the described delay mechanisms, is added to HiSIM-QS [4]. The improved circuit-simulation model is called HiSIM-NQS in the following. Simulation results of the transient drain current for rise times of 80ps as well as 20ps are plotted in Figs. 5a and b, respectively. The artifacts associated with conventional QS modeling, which increase for faster switching as depicted by the circles, are successfully eliminated.

Fig. 6 shows that HD for a $1 \mathrm{KHz}$ input is correctly modeled with HiSIM-QS. For the calculation, only measured $I-V$ characteristics are fitted, and no extra fitting parameters are required even for the higher-order distortion calculation. This validates the model at low frequency operation. At low drain bias and low frequency, the HD curves are directly related to the carrier mobility. Figs. 7a and b show the $1 \mathrm{KHz} \mathrm{HD}$ curves and the corresponding mobility model with its derivatives, respectively. At higher frequencies, two new features appear. One is a shift of the harmonic curves as shown in Fig. 8 for $5 \mathrm{GHz}$ switching. This shift is most obvious at low gate bias, where the cut-off frequency is comparable with the fundamental harmonic frequency $\omega_{0}$. The second feature is a flattening of the first harmonic (HD1), as shown in Fig. 9a, due to rising values at low gate bias. This can be understood by the fact that the transient drain current consists of two components, the conductive current and the channel-charging current. Under high switching frequency, the channel-charging current increases and becomes large in comparison to the conductive current for low gate bias.

At $5 \mathrm{GHz}$, the first harmonic changes only slightly as a function of mobility as verified in Fig. 9b. This means that the transport of carriers in the channel at high frequency is no longer dominated by the carrier mobility as usual in the DC and low frequency cases. The charging of the channel itself determines the carrier response under high frequency operation. Therefore, correct modeling of channel charging is essential, especially for NQS circuit-simulation models. Although HiSIM-NQS results for high-frequency HD 
are in qualitative agreement with previously published measurements for a different technology [2], precise measurements of GHz MOSFET operation are still necessary to further validate these results.

\section{Conclusion}

The modeling of non-quasi-static (NQS) MOSFET behavior based on the time-delay of the carrier response, as applied for the circuit-simulation model HiSIM-NQS, has been described. Computational runtime increase with implemented carrier dynamics is only $7 \%$ in comparison to HiSIM-QS. This computationally efficient modeling is already sufficient for correct reproduction of transient drain current as well as harmonic distortion characteristics.

\section{References}

[1] Y. Tsividis, et al., "Harmonic Distortion in Single-Channel MOS Integrated Circuits," IEEE Solid-State Circuits, Vol. SC-16, No. 6, pp. 694-702, Dec. 1981.

[2] R. van Langevelde, et al., "RF Applications of MOS Model 11," Proc. Modeling and Simulation of Microsystems, pp. 674-677, 2002.

[3] L.-J. Pu, et al., "Harmonic Distortion of the Four-Terminal MOSFET in Non-Quasistatic Operation," IEE Proceedings, Vol. 137, Pt.G, No. 5, pp. 325-332, Oct. 1990.

[4] http://www.starc.or.jp/kaihatu/pdgr/hisim/index.html

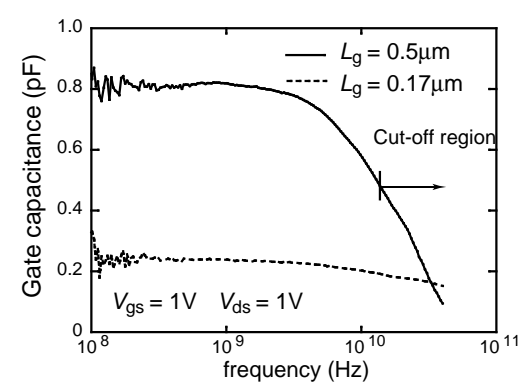

Figure 1: Measured reduction of the MOSFET gate capacitance as a function of input signal frequency for a pocket-implant technology.

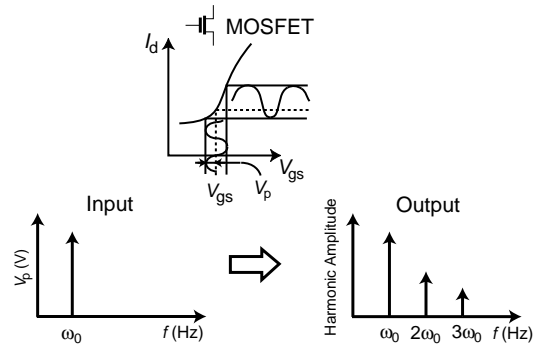

Figure 3: Schematic explanation of harmonic distortion (HD).

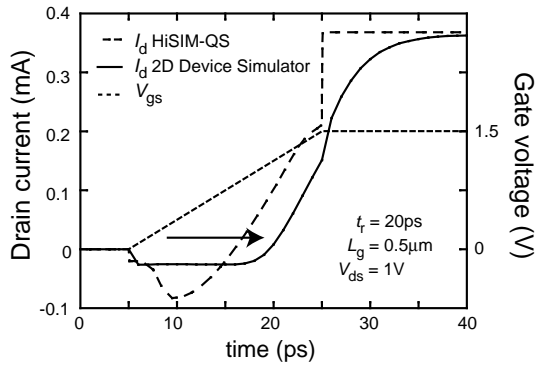

Figure 2: Delay of current flow under RF switching.

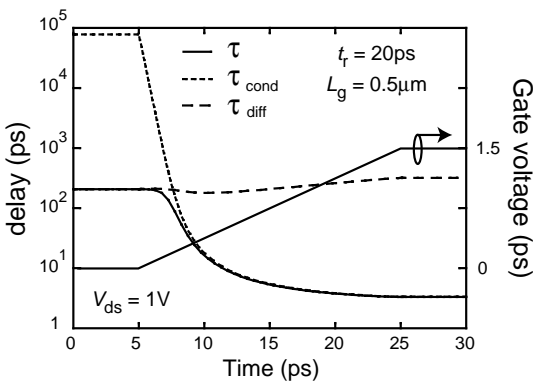

Figure 4: Time-delay mechanisms employed in HiSIM-NQS. Plotted is the transient $\tau$ for 20ps switching at high drain bias $\left(V_{\mathrm{ds}}=1 \mathrm{~V}\right)$. 

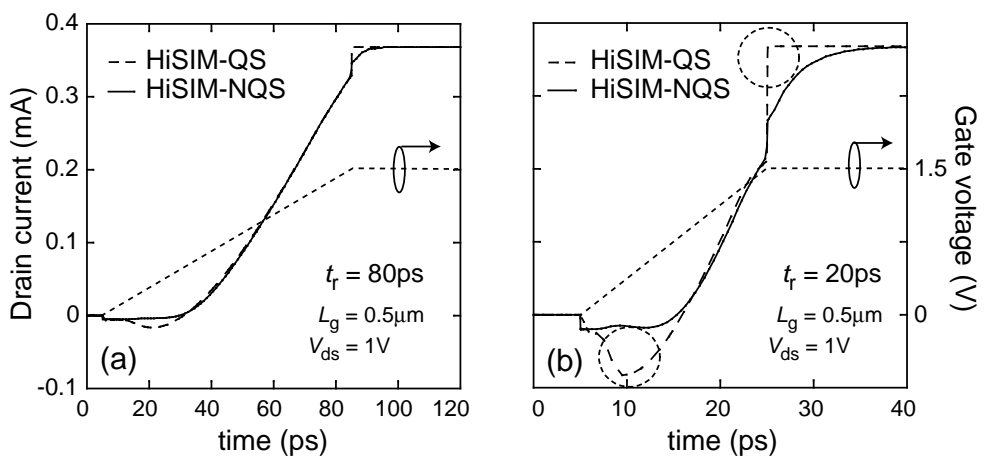

Figure 5: Elimination of QS artifacts in the transient drain current for (a) 80ps and (b) 20ps switching time, due to the described NQS-modeling approach.

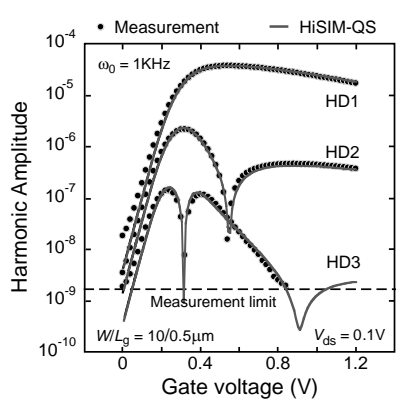

Figure 6: Reproduction of harmonic distortion characteristics at $1 \mathrm{KHz}$.

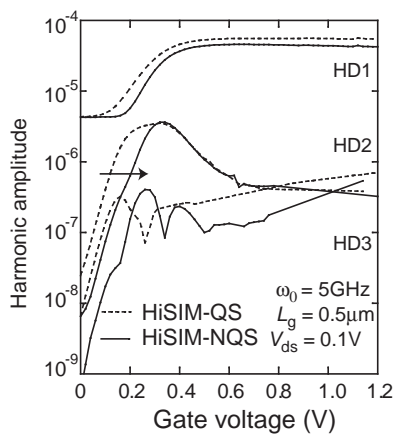

Figure 8: Harmonic distortion characteristics at high frequency of $5 \mathrm{GHz}$.

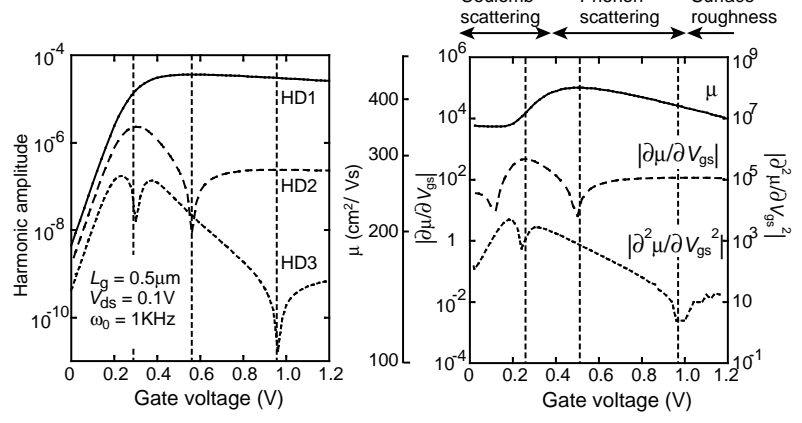

Figure 7: (a) Simulated harmonic distortion characteristics at low drain bias and low frequency $(1 \mathrm{KHz})$. (b) Mobility model of HiSIM and its derivatives.

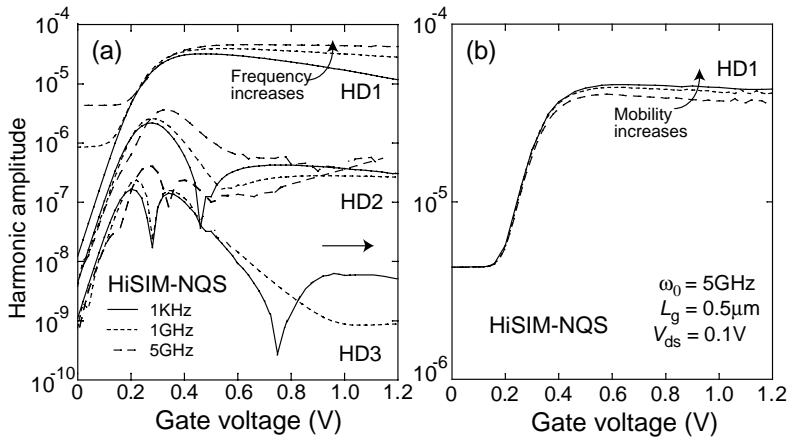

Figure 9: HD characteristics at different frequencies. (a) The increase of HD1 at low gate bias is attributed to the increasing effect of the channel-charging current. (b) Only a slight change of HD1 results, if the mobility (surface roughness) is changed. 